\title{
Resident Physician Wellness Curriculum: A Study of Efficacy and Satisfaction
}

\author{
Dennis Lefebvre ${ }^{1}$, Kathryn A. Dong ${ }^{2}$, Erica Dance ${ }^{1}$, Rhonda J. Rosychuk ${ }^{3}$, Mark Yarema ${ }^{4}$, Danielle
} Blouin ${ }^{5}$, Jennifer Williams ${ }^{6}$, Brian H. Rowe ${ }^{7}$

\begin{abstract}
1. Emergency Medicine, University of Alberta, Edmonton, CAN 2. Emergency Medicine, Addiction Medicine, University of Alberta, Edmonton, CAN 3. Pediatrics, University of Alberta, Edmonton, CAN 4. Emergency Medicine, University of Calgary, Calgary, CAN 5. Emergency Medicine, Kingston Health Sciences Centre / Queen's University, Kingston, CAN 6. Emergency Medicine, University of British Columbia, Kelowna, CAN 7. Emergency Medicine, School of Community Based Medicine, University of Alberta, Edmonton, CAN
\end{abstract}

$\square$ Corresponding author: Dennis Lefebvre, dclefebvre@gmail.com

Disclosures can be found in Additional Information at the end of the article

\section{Abstract}

\section{Background}

Recent literature highlights the alarming prevalence of burnout, depression, and illness during residency training; a trend that is also linked to suboptimal patient care. Dedicated wellness curricula may be one solution to this concerning issue.

\section{Purpose}

To determine the effect of a multi-faceted wellness curriculum during emergency medicine residency training on wellness scores and to assess resident satisfaction with the program.

\section{Methods}

This study was conducted via a longitudinal survey. In 2009, a faculty-derived resident wellness curriculum (F-RWC) was initiated. This program was then bolstered with a parallel resident-derived curriculum (RRWC) one year later, in 2010. Emergency medicine residents were surveyed in 2009, 2010, and 2011 to assess wellness at baseline, after one year of the F-RWC, and after one year of combined RWCs, respectively. Surveys included two validated assessment instruments (the Brief Resident Wellness Profile (BRWP) and the SF- $8^{\mathrm{TM}}$ Health Survey), a satisfaction Likert scale, and a demographics information sheet.

Received 06/27/2019 Review began 07/08/2019 Review ended 08/01/2019 Published 08/03/2019

() Copyright 2019

Lefebvre et al. This is an open access article distributed under the terms of the Creative Commons Attribution License CCBY 3.0., which permits unrestricted use, distribution, and reproduction in any medium, provided the original author and source are credited.

\section{Results}

The survey response rates were $89 \%(n=17), 100 \%(n=17)$, and $83 \%(n=24)$ from 2009, 2010, and 2011, respectively, for a total of 58 participants. From baseline in 2009, there was a significant improvement in resident wellness, with the addition of parallel RWC by 2011, as measured by the BRWP ( $\mathrm{p}=0.024)$. The faces scale, a subset of the BRWP, showed a trend toward benefit but did not reach statistical significance $(p=0.085)$. There was no evidence of a statistically significant change in $\mathrm{SF}-8^{\mathrm{TM}}$ scores over time. Participants consistently reported positive satisfaction scores with RWC initiatives.

\section{Conclusions}

Dedicated RWC, with input from both faculty and resident physicians, improved wellness during residency training with a high degree of participant satisfaction. Such programs are needed to support resident physicians during their training.

Categories: Emergency Medicine, Medical Education

Keywords: wellness, physician well-being, physician burnout, resident wellness, resident training, resident curriculum

\section{Introduction}

It is well-established that resident physician training, or "residency," is a challenging period in the career path of physicians. Many authors have characterized the morbidity of a struggling physician in terms of "burnout," a quantifiable psychologic syndrome composed of emotional exhaustion, depersonalization, and reduced personal accomplishment [1]. Others have highlighted alarming rates of depression [2-3], suicide [4], as well as relationship peril [5] and sexual dysfunction [6], attributed to residency training. Resident burnout is pervasive across various resident subspecialties and has been linked to suboptimal patient care and 
medical error [7].

In the recent past, many hypothesized that burnout issues were born from excessive resident duty hours. In response, duty hours were shortened and regulated in the United States [8], Europe [9], and Canada [10] in 2003, 2009, and 2010, respectively. The subsequent analysis of duty hours reform, however, revealed no improvement in sleep hours, work hours, medication errors, or depression [11]. Moreover, despite its good intentions, duty hours reform has also been met with criticism over concern for adequate training experience and compromised patient care [12].

Recently, some authors have advocated for a new approach to addressing resident burnout: dedicated wellness curricula during residency training [7,13]. Resident wellness curricula (RWC) aim to add support to residents rather than detract from hours of experience. Ideally, the support offered in an RWC should include initiatives that are both active and passive, preventive and responsive, and address the spheres of physical, mental, educational, social, financial, and lifestyle wellness [7,14-15]. Given that RWC are a relatively new concept, there is currently a lack of literature demonstrating efficacy with validated instruments. To date, most of the evidence for wellness initiatives is derived from mindfulness education sessions initiated by the researchers themselves [16-17]. We hypothesized that a robust RWC, with parallel input from both faculty and the resident body, would lead to a significant improvement in resident wellness measurable with a validated scoring tool, the Brief Resident Wellness Profile (BRWP) [18] and/or the SF-8TM Health Survey [19].

\section{Materials And Methods}

In Canada, dedicated Emergency Medicine residency training is accredited by the Royal College of Physicians and Surgeons. To achieve fellowship designation, trainees must complete a five-year, postgraduate residency program at a designated university. This training includes an initial basic clinical training year with mixed rotations through surgery, obstetrics, internal medicine, anesthesia, and so forth. Subsequent training years include rotations in intensive care with increasing time spent in the Emergency Department. Senior residents in years four and five practice almost exclusively in emergency medicine.

This study was reviewed and approved by the Research Ethics Office at the University of Alberta (Pro00024292). Subsequent to ethics approval, Royal College resident physicians in the Department of Emergency Medicine were hand-delivered surveys at scheduled academic events in 2009, 2010, and 2011. These dates corresponded to a baseline (no RWC) assessment, one year post-exposure to a faculty-derived RWC (F-RWC), and one year post-exposure to parallel faculty and resident-derived RWC (R-RWC). Of note, the 2009 and 2010 groups were identical, however, owing to both graduation and recruitment, the 2011 group had some new participants and slightly larger sample size. Residents not in attendance had surveys distributed to their individual hospital mailboxes. Survey completion was voluntary, and consent was implied with the confidential return of sealed surveys to the Program Administrator. Participant information was not linked across all three time points. Surveys were held in a locked cabinet in the Department of Emergency Medicine until the time of data analysis after 2011.

Survey packages included the Brief Resident Wellness Profile (BRWP) with faces scale [18], the SF-8 $8^{\mathrm{TM}}$ Health Survey [19], and a demographics section. The 2011 cohort also received a satisfaction survey composed of a seven-point Likert scale for each of the RWC initiatives completed in the year prior. In addition, participants were invited to leave comments in a text box to further describe their degree of satisfaction with the program.

The F-RWC was developed and implemented by two Emergency Physicians who were educators in the resident training program. The F-RWC consisted of the following: 1) biannual, confidential, one-on-one meetings between each resident and a dedicated faculty wellness mentor; and 2) wellness-related lectures added to mandatory academic rounds. The goal of the one-on-one meetings was to identify and intervene if symptoms of burnout and depression, problematic substance use, relationship issues, and financial concerns and/or challenges in the hospital setting were present. Each session was scheduled for one hour and residents were able to schedule more meetings with the wellness mentor if needed or desired. The wellness mentors were also available throughout the year for urgent wellness-related concerns. Grand Rounds topics included fitness, healthy food preparation, and financial planning.

The R-RWC was developed and implemented by a senior resident in the Department of Emergency Medicine as part of an academic research initiative. The R-RWC was targeted to specific wellness domains and consisted of the following:

1) Educational Wellness: post-graduate year (PGY)-1 “on-call survival booklet," PGY-2 exam binders to streamline preparation for the Medical Council of Canada Qualifying Exam Part II, PGY-3 primer booklet for the eight-week intensive care unit (ICU) rotation, and a toolkit for all residents, including drug reference booklet, reference cards, and pocket light. 


\section{Cureus}

2) Social Wellness: monthly or semi-monthly organized outings to local professional sports teams, live music, or dinner engagements.

3) Mental Wellness: monthly new music playlists for exercise or study, wellness seminar at the annual resident retreat, Christmas gift donation drive, blood donation drive, and Remembrance day perspective material.

4) Financial Wellness: access to income tax software, funding for travel to the annual resident retreat, and coupons to local shops.

5) Lifestyle Wellness: healthy and easy recipes for home-cooked meals, a non-medical library with recent novels and magazines.

6) Physical Wellness: 24-hour access to complimentary bottled water and sports drinks in the resident lounge.

Data analysis included descriptive statistics (e.g., mean, standard deviation (SD), frequencies, and percents) for demographic variables and outcomes by year. Mixed-effects linear regression models were used to assess the effect of year on continuous outcomes (random effect for the subject as the same subjects were in the first two years). Additional explanatory variables (age, sex, marital status, debt amount, debt indicator, and moonlight) were also added to examine the adjusted effect of year. Fisher's exact (FE) test assessed the association between faces scores and year. P-value (p) less than 0.05 was considered statistically significant. Analyses were performed in R Core Team (2016) [20].

\section{Results}

\section{Resident demographics}

Anonymized information on residents in the program is depicted in Table 1 . There was a total of 58 participants in this study with a slight predominance of males (62\%). The ages were similar among groups with a mean age of $30.65,31.65$, and 30.17 years for 2009,2010 , and 2011 , respectively. Other demographic variables, including marital status, children, debt, and frequency of moonlighting shifts, also remained similar over the three time points (Table 1). All subjects were emergency medicine residents from the University of Alberta, Canada. 


\section{Cureus}

\begin{tabular}{|c|c|c|c|}
\hline & 2009 & 2010 & 2011 \\
\hline \multicolumn{4}{|l|}{ Sex } \\
\hline Male & 12 & 12 & 12 \\
\hline Female & 5 & 5 & 11 \\
\hline Missing & 0 & 0 & 1 \\
\hline Total & 17 & 17 & 24 \\
\hline \multicolumn{4}{|l|}{ Age } \\
\hline Mean (standard deviation) & $30.65(2.12)$ & $31.65(2.12)$ & $30.17(2.94)$ \\
\hline \multicolumn{4}{|l|}{ Marital Status } \\
\hline Married or Common Law & 6 & 6 & 9 \\
\hline Dating & 7 & 7 & 4 \\
\hline Divorced or Widowed & 0 & 0 & 0 \\
\hline Single & 4 & 4 & 10 \\
\hline Missing & 0 & 0 & 1 \\
\hline \multicolumn{4}{|l|}{ Children } \\
\hline Yes & 0 & 0 & 2 \\
\hline No & 17 & 17 & 22 \\
\hline Missing & 0 & 0 & 0 \\
\hline \multicolumn{4}{|l|}{ Debt Amount (CAD) } \\
\hline$<\$ 10,000$ in debt & 3 & 3 & 3 \\
\hline$\$ 10,000-\$ 99,999$ & 8 & 7 & 10 \\
\hline$\$ 100,000-\$ 199,999$ & 6 & 7 & 5 \\
\hline$\geq \$ 200,000$ & 0 & 0 & 4 \\
\hline \multicolumn{4}{|l|}{ IViouringnit } \\
\hline Never & 6 & 5 & 6 \\
\hline < once per month & 2 & 1 & 3 \\
\hline one shift per month & 6 & 8 & 6 \\
\hline 2-4 shifts per month & 3 & 3 & 6 \\
\hline$>4$ shifts per month & 0 & 0 & 1 \\
\hline Missing & 0 & 0 & 1 \\
\hline
\end{tabular}

TABLE 1: Demographic information by cohort year

\section{Survey response}

The response rates for surveys completed in 2009, 2010, and 2011 were $89 \%(\mathrm{n}=17), 100 \%(\mathrm{n}=17)$, and $83 \%$ $(\mathrm{n}=24)$, respectively.

\section{Wellness scores}

Mean scores for the Brief Resident Wellness Profile (BRWP) in 2009 (baseline), 2010 (one year after Facultyderived Resident Wellness Curriculum; F-RWC), and 2011 (one year after combined F-RWC and Resident- 


\section{Cureus}

derived Resident Wellness Curriculum; R-RWC) were 18.94, 20.69, and 21.12 ( $\mathrm{p}=0.024)$, respectively, demonstrating a significant improvement in resident wellness after exposure to the combined RWC from 2009 to 2011 (Figure 1A). Even when adjusted for other factors in a multivariable mixed model (see the methods section), the BRWP remained different over the years $(\mathrm{p}=0.011)$. There was no evidence of a statistically significant change over time measured by the SF-8TM Health Survey (Figures $1 B-1 C$ ) in either the Physical Component Summary (PCS-8; KW test, $\mathrm{p}=0.174$ ) or the Mental Component Summary (MCS-8; KW test, $\mathrm{p}=0.392$ ).
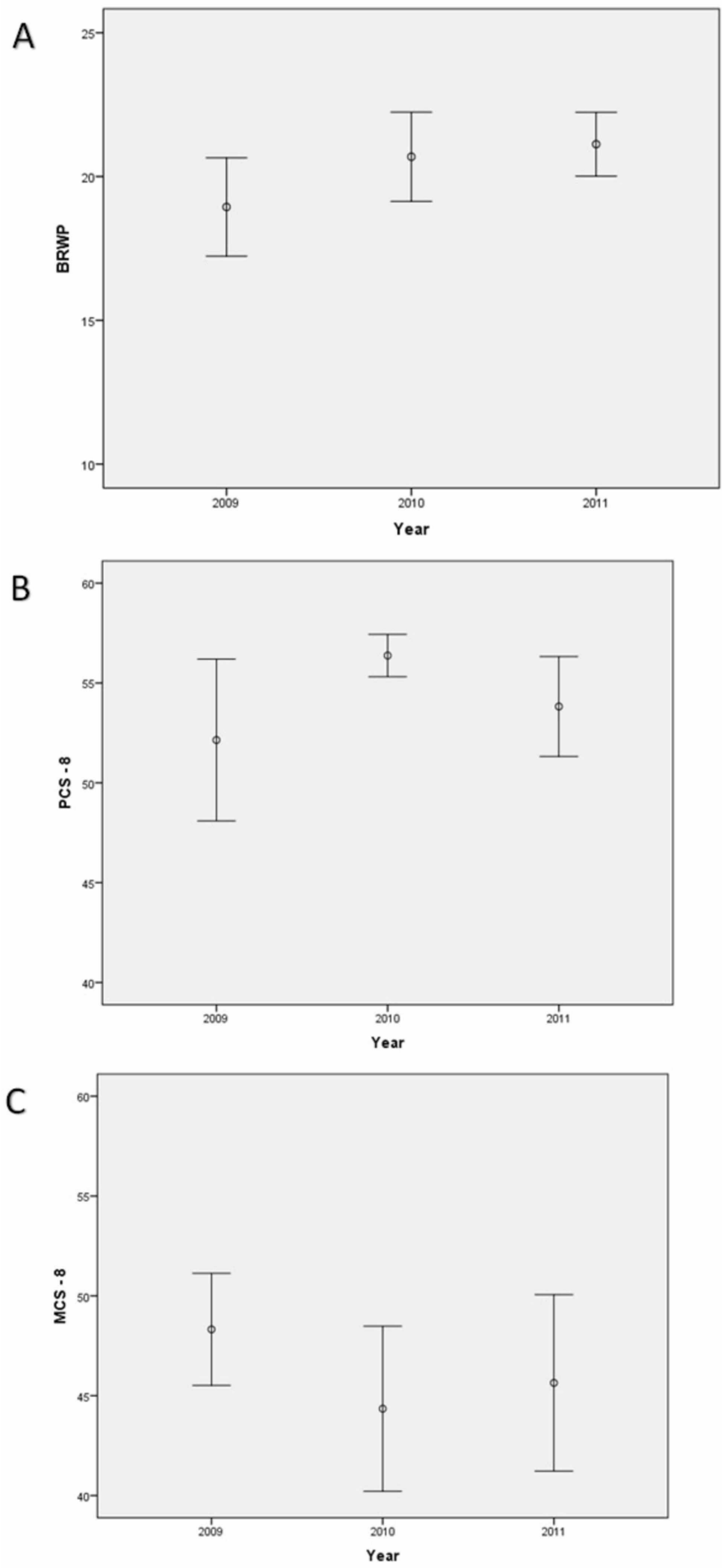

\section{FIGURE 1: Wellness scores}

A) Scores from the Brief Resident Wellness Profile (BRWP; error bars represent mean and 95\% confidence interval for each year). There was a significant improvement over time from 2009 to 2011 ( $p=0.024)$; B) Scores from the SF-8 health survey, Physical Component Summary (PCS-8; $p=0.174$ ); C) Scores from the SF-8 health survey, Mental Component Summary (MCS-8; $\mathrm{p}=0.392$ ). 


\section{Cureus}

\section{Faces scores}

This subset of the BRWP tended to be higher for 2011 than 2009 (Figure 2), suggesting a trend toward improvement, however, there was no evidence of an association between the faces score and year (FE test, $\mathrm{p}=0.085$ ).

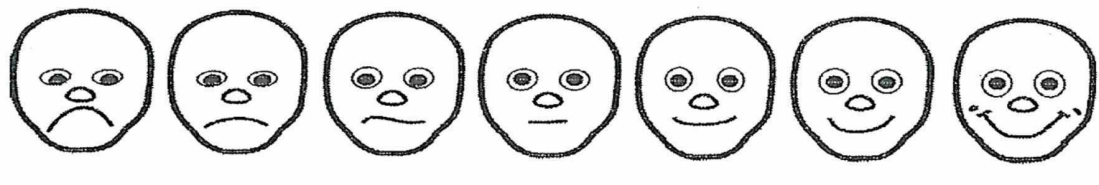

$=3$

$=4$

$=6$

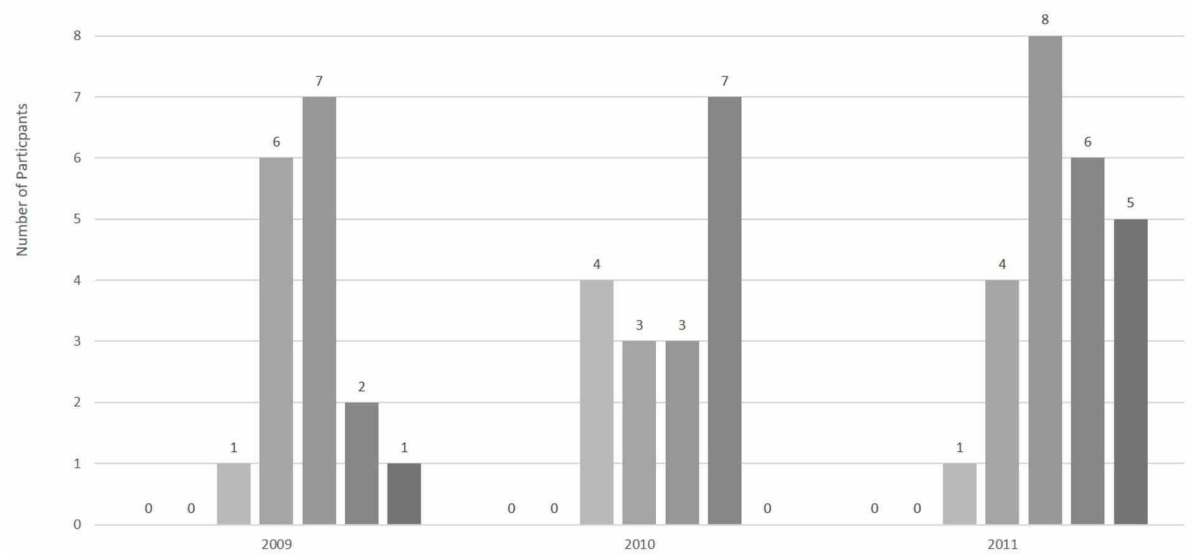

FIGURE 2: Faces scores

Faces scores (range from most unhappy face $=0$, to happiest face $=6$ ). The Fisher exact test showed improvement from 3.765 to 4.417 (from 2009 to 2011) but did not reach statistical significance $(p=0.084)$. Faces illustration from Keim et al. 2006 [18].

\section{Program evaluation}

Resident participation and satisfaction with the R-RWC were assessed in 2011 using a seven-point Likert scale (Figure 3). Each survey organized and listed R-RWC interventions under the five domains of wellness (educational, social, mental, financial, lifestyle, and physical) to enhance recall. There were a total of 282 participants at various R-RWC events, and 272/282 (96\%) indicated a slightly positive, positive, or strongly positive experience. Further, 151/282 (54\%) reported a strongly positive satisfaction score. Mental wellness initiatives (see the methods section) were the best-attended events.

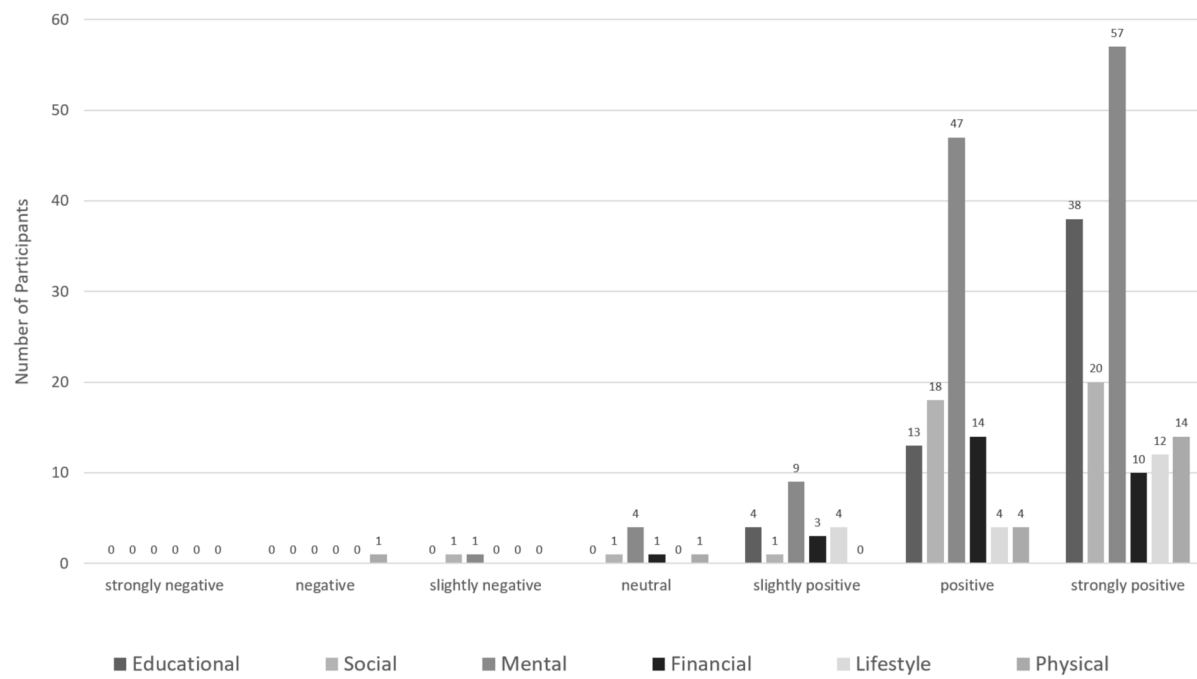




\section{Discussion}

Residency training is a stressful period for learners, and recent literature demonstrates the consequences of these risks and the steps taken to address them. In a landmark case, the death of Libby Zion in 1984 revealed a potential link between resident burnout and patient safety [21]. Over the next 20 years, researchers consistently published alarming rates of burnout [22-26], depression [2-3], morbidity [27], and mortality [4] among resident physicians. Many hypothesized that the main culprit was excessive duty hours during residency training. However, multinational regulations of duty hours [8-10] in the 2000s failed to alleviate the substantial risks associated with training [28] and were subsequently met with widespread criticism from educators [12].

Since modification of duty hours alone has not eliminated the development of burnout, additional interventions are required to address this issue. The current study adds to a growing body of literature suggesting that dedicated wellness training is beneficial to resident physicians and the patients they treat $[7,12,16]$. To our knowledge, this is the first study to investigate the efficiency and satisfaction of a multifaceted RWC using a validated tool tailored specifically for resident physicians. Unique to our program is the combination of both faculty and resident input, the targeting of multiple wellness domains, and the use of both active and passive interventions.

The key finding in our study is that the combination of F-RWC and R-RWC over a two-year period led to a significant benefit in resident wellness as measured by the BRWP. During this period, no other major interventions were adopted or implemented that could explain these changes. Moreover, duty hours and residency rotations were unchanged. Of note, no evidence of a statistically significant benefit seen with FRWC alone that may suggest that input from the resident population (the target audience) was critically important. However, this outcome could also be the result of the relatively low sample size.

There was no evidence of a statistical benefit seen in physical and/or mental health as measured by the SF8TM Health Survey. This instrument provides a generic measure of health that is not specific to any particular population. Conversely, resident physicians are a niche population, of similar age, with stressors that are unique to this profession. This is likely the reason that a benefit was seen with a tailored resident wellness instrument (BRWP) as opposed to the non-specific SF-8TM.

A secondary finding in our analysis was the high level of resident satisfaction with the program. This indicates that not only does our RWC improve wellness, but it is also well-liked by the target audience. Written comments from residents were consistently positive. Some examples from the satisfaction survey included: "Fantastic, made me feel like someone cared about my success both at work and away from work," "Prevents the degree of separation that naturally occurs between staff and residents," and "I think it is very positive, especially for new residents joining the group for support and sense of community.”

The strengths of our study include the multi-faceted nature of the program, the use of published and validated instruments to measure resident physician wellness (BRWP) and general health (SF-8TM), the measurement of wellness at three different time points, and the high survey response rate. There are also some limitations to this study. First, the study population is small (total of 58 participants) and only includes residents in the Emergency Medicine training program from a single institution. Second, the cross-sectional format of this study means that cohorts from 2009, 2010, and 2011 had different resident populations with variable exposures to our RWC. This is due to resident turnover, as each year graduating residents become staff, and graduating medical students begin their residency training. Third, owing to small cohort sizes, there were not enough participants to include a parallel control group over the three time points. Finally, due to the need for anonymity, details about non-responders and individual changes over time were not able to be analyzed.

In conclusion, this small, single-center study shows that dedicated, multi-faceted RWC with input from both faculty and resident physicians can provide a significant wellness benefit to a resident population that strongly supports the program. Future research is needed to assess similar curricula in larger resident populations, in other subspecialties, and at other institutions. These programs are effective, well-liked, wellattended, and are designed to minimally interfere with formal resident education or duty hours.

\section{Conclusions}

Residency training is a challenging period in the development of future physicians. For some, the demands and stressors of training can lead to burnout or worse. To date, putative solutions to this issue, such as duty 
hours reform, have shown to be ineffective. In response, a novel approach is an introduction of dedicated Resident Wellness Curricula (RWC) to residency training. Here, dedicated RWC with input from both faculty and resident physicians improved wellness during residency training with a high degree of participant satisfaction. Such programs are needed to support resident physicians during their training.

\section{Additional Information \\ Disclosures}

Human subjects: Consent was obtained by all participants in this study. Research Ethics Office at the University of Alberta issued approval Pro00024292. This longitudinal survey study was reviewed and approved by the Research Ethics Office at the University of Alberta (Pro00024292). Animal subjects: All authors have confirmed that this study did not involve animal subjects or tissue. Conflicts of interest: In compliance with the ICMJE uniform disclosure form, all authors declare the following: Payment/services info: Dr. Lefebvre reports grants from Canadian Association of Emergency Medicine, grants from Canadian Institutes of Health Research, during the conduct of the study; other from Canadian Association of Emergency Medicine, grants from Canadian Institutes of Health Research, outside the submitted work. Financial relationships: All authors have declared that they have no financial relationships at present or within the previous three years with any organizations that might have an interest in the submitted work. Other relationships: All authors have declared that there are no other relationships or activities that could appear to have influenced the submitted work.

\section{Acknowledgements}

The authors would like to thank the residents in the Emergency Medicine training program at the University of Alberta for their participation in this program. In addition, we would like to thank the staff for their participation as mentors and advisors, and Iram Usman, M.Sc., at the University of Alberta, for assistance with data analysis. This F-RWC portion of the study was funded by the Canadian Association of Emergency Physicians. Dr. Rowe's research is supported by the CIHR as a Tier I Canada Research Chair in Evidencebased Emergency Medicine from the Government of Canada (Ottawa, ON). These funding organizations had no involvement in any aspect of the conduct, analysis, and manuscript preparation of this study; CIHR takes no responsibility for the conduct or results of this review.

\section{References}

1. Evaluating Stress: A Book of Resources, Third Edition . Zalaquett CP, Wood RJ (ed): Scarecrow Press, New York; 1997. 10.1017/S0033291798257163

2. Peterlini M, Tiberio IF, Saadeh A, Pereira JCR, Martins MA: Anxiety and depression in the first year of medical residency training. Med Educ. 2002, 36:66-72. 10.1046/j.1365-2923.2002.01104.x

3. Rosen IM, Gimotty PA, Shea JA, Bellini L: Evolution of sleep quantity, sleep deprivation, mood disturbances, empathy, and burnout among interns. Acad Med. 2006, 81:82-85. 10.1097/00001888-200601000-00020

4. Williams LS: Manitoba suicides force consideration of stresses facing medical residents . CMAJ. 1997, 156:1599-1602.

5. Koran LM, Litt IF: House staff well-being. West J Med. 1988, 148:97-101.

6. Sangi-Haghpeykar H, Ambani D, Carson SA: Stress, workload and sexual dysfunction among physician residents in training. Int J Clin Pract. 2009, 63:462-7. 10.1111/j.1742-1241.2008.01845.x

7. Lefebvre DC: Perspective: resident physician wellness: a new hope . Acad Med. 2012, 87:598-602. 10.1097/ACM.0b013e31824d47ff

8. Accreditation Council for Graduate Medical Education. History of duty hours . Accessed: June 20, 2019: http://www.acgme.org/What-We-Do/Accreditation/Duty-Hours/History-of-Duty-Hours.

9. Eggertson L: Residents claim 24-hour call violates Charter rights . CMAJ. 2009, 180:918. 10.1503/cmaj.090527

10. What is the European working time directive? (2009). Accessed: June 20, 2019: http://webarchive.nationalarchives.gov.uk/20100330181623/http://www.dh.gov.uk/en/Managingyourorganisation/Wo

11. Landrigan CP, Fahrenkopf AM, Lewin D, et al.: Effects of the Accreditation Council for Graduate Medical Education duty hour limits on sleep, work hours, and safety. Pediatrics. 2008, 122:250-258. 10.1542/peds.2007-2306

12. Millard WB: For whom the bell commission tolls: unintended effects of limiting residents' hours . Ann Emerg Med. 2009, 54:25-29. 10.1016/j.annemergmed.2009.08.008

13. McClafferty H, Brown OW: Physician health and wellness. Pediatrics. 2014, 134:830-835. 10.1542/peds.2014-2278

14. The CanMEDS 2005 Physician Competency Framework. Better Standards. Better Physicians. Better Care. Ottawa. Frank JR (ed): The Royal College of Physicians and Surgeons of Canada, Ontario, Canada; 2005.

15. VanDewark K: CanMEDS Physician Health Guide: A Practical Handbook for Physician Health and WellBeing. Puddester D, Flynn L, Cohen J. (ed): The Royal College of Physicians and Surgeons of Canada, Ottawa; 2009.

16. Krasner MS, Epstein RM, Beckman H, Suchman AL, Chapman B, Mooney CJ, Quill TE: Association of an educational program in mindful communication with burnout, empathy, and attitudes among primary care physicians. JAMA. 2009, 302:1284-1293. 10.1001/jama.2009.1384

17. Goldhagen BE, Kingsolver K, Stinnett SS, Rosdahl JA: Stress and burnout in residents: impact of 


\section{Cureus}

mindfulness-based resilience training. Adv Med Educ Pract. 2015, 6:525-532. 10.2147/AMEP.S88580

18. Keim SM, Mays MZ, Williams JM, Serido J, Harris RB: Measuring wellness among resident physicians . Med Teach. 2006, 28:370-374. 10.1080/01421590600625320

19. Ware JE: How to Score and Interpret Single-Item Health Status Measures: A Manual for Users of the SF-8 Health Survey : (With a Supplement on the SF-6 Health Survey). QualityMetric, Inc., Boston, MA; 2001.

20. The R project for statistical computing . (2016). https://www.r-project.org/.

21. Goitein L: The girl who died twice: every patient's nightmare: the Libby Zion case and the hidden hazards of hospitals. N Engl J Med. 1996, 334:201-202. 10.1056/NEJM199601183340323

22. Shanafelt TD, Bradley KA, Wipf JE, Back AL: Burnout and self-reported patient care in an internal medicine residency program. Ann Intern Med. 2002, 136:358-367. 10.7326/0003-4819-136-5-200203050-00008

23. Becker JL, Magdy MP, Klock SC: Burnout, depression, and career satisfaction: cross-sectional study of obstetrics and gynecology residents. Am J Obstet Gynecol. 2006, 195:1444-1449. 10.1016/j.ajog.2006.06.075

24. Fahrenkopf AM, Sectish TC, Barger LK, et al.: Rates of medication errors among depressed and burnt out residents: prospective cohort study. BMJ. 2008, 336:488-491. 10.1136/bmj.39469.763218.BE

25. Golub JS, Weiss PS, Ramesh AK, Ossoff R, Michael Johns M: Burnout in residents of otolaryngology-head and neck surgery: a national inquiry into the health of residency training. Acad Med. 2007, 82:596-601. 10.1097/ACM.0b013e3180556825

26. Martini M, Arfken CL, Churchill A, Balon R: Burnout comparison among residents in different medical specialties. Acad Psychiatry. 2004, 28:240-242. 10.1176/appi.ap.28.3.240

27. Clever LH: Who is sicker: patients - or residents? Residents' distress and the care of patients . Ann Intern Med. 2002, 136:391-393. 10.7326/0003-4819-136-5-200203050-00012

28. Iglehart JK: Revisiting duty-hour limits - IOM recommendations for patient safety and resident education . N Engl J Med. 2008, 359:2633-2635. 10.1056/NEJMp0808736 